\title{
Vulnerabilidad y Exigibilidad de Derechos: la Perspectiva de Niños, Niñas y Adolescentes
}

\section{Vulnerability and Rights Enforceability: the Perspective of Children and Adolescents}

\author{
María Malena Lenta y Graciela Zaldúa \\ Universidad de Buenos Aires
}

\begin{abstract}
Se presenta un estudio de casos múltiple, constituido por casos típicos de vulneración de derechos, en el que cada uno de los 16 niños, niñas o adolescentes participantes constituyó un caso. Desde un enfoque de psicología social comunitaria, se analizaron los sentidos y significaciones que construyen niños, niñas y adolescentes en territorios de vulnerabilidad psicosocial, en torno a su vida cotidiana y a su situación de acceso a derechos. Se desarrolló un estudio exploratorio-descriptivo con enfoque cualitativo y se implementaron relatos de vida, grupos focales y observación naturalista. Para el análisis de la información se utilizó una estrategia de enfoque biográfico. Los resultados se organizaron según tres dimensiones del relato biográfico: la realidad socio histórica vinculada a la formación económico social, la realidad psíquica focalizada a las dimensiones del ser y el hacer de los participantes y la realidad discursiva como instancia de análisis retrospectivo y prospectivo del vínculo con los otros. Los casos presentados cuestionaron la eficacia de las políticas sociales de restitución de derechos. Las trayectorias de vida señalaron obstáculos micro y macro-políticos para el acceso a derechos, que sostienen la reproducción social de las desigualdades y tienen efectos desubjetivantes. Las situaciones que garantizan apuntalamientos identificatorios y la exigibilidad de derechos promueven procesos subjetivantes individuales y colectivos de los actores involucrados y proponen nuevos desafíos en la construcción del proyecto de vida.
\end{abstract}

Palabras clave: psicología social comunitaria, niñez, trayectorias de vida, políticas sociales

\begin{abstract}
A multiple-case study is presented, comprising typical cases of rights violations where each of the 16 child and adolescent participants constituted a case. Adopting a social community psychology approach, the authors analyzed the meanings that children and adolescents living in territories marked by psychosocial vulnerability construct with respect to their everyday life and their access to rights. A descriptive-exploratory study of a qualitative nature was conducted. Life stories, focus groups, and naturalistic observation were implemented. A biographical strategy was adopted to analyze the information collected. Results were organized according to three dimensions of biographical narratives: sociohistorical reality, linked to socioeconomic education; psychic reality, focused on the participants' being and doing dimensions; and discursive reality, as an instance of retrospective and prospective analysis of the connection with others. The cases presented called into question the effectiveness of social policies aimed at restoring people's rights. The participants' life trajectories revealed micro- and macro-political obstacles to accessing rights, a situation that supports the social reproduction of inequalities and has de-subjectivizing effects. Situations that guarantee identity grounding and rights enforceability promote individual and collective subjectivizing processes in the actors involved and pose new challenges in the construction of one's life project.
\end{abstract}

Keywords: social community psychology, childhood, life trajectories, social policies

Para la psicología social comunitaria, en tanto campo disciplinar abierto que interpela los procesos psicosociales de opresión y exclusión (Montero, 2006; Zaldúa \& Pawlowicz, 2011), la vulnerabilidad resulta una categoría clave. Desde este campo, su definición no se circunscribe a la descripción de la pobreza en tanto insuficiencia de recursos materiales para salir del plano de la mera supervivencia, sino que alude a un conjunto de vínculos frágiles que los sujetos, los grupos y las comunidades desarrollan en relación al trabajo, a las relaciones sociales y a las capacidades de agencia, es decir, al ejercicio de la ciudadanía a través de la vida política (Zaldúa, Pawlowicz, Longo, Sopransi \& Lenta, 2016).

Butler (2004/2012) hace referencia a vulnerabilidad como condición constitutiva de todo ser humano que lo compele al desarrollo de la vida política para resguardarse del estado de indefensión originaria al ser-lanzados-al-mundo. Sin embargo, añade que la vulnerabilidad se exacerba bajo ciertas condiciones sociales y políticas. La exposición a la violencia y al sufrimiento tiene una distribución diferencial a lo largo

María Malena Lenta y Graciela Zaldúa, Facultad de Psicología, Universidad de Buenos Aires.

La correspondencia relativa a este artículo debe ser dirigida a María Malena Lenta, Facultad de Psicología, Universidad de Buenos Aires, Independencia 3065, Código Postal 1221, Ciudad Autónoma de Buenos Aires, Argentina. E-mail: malenalenta@psi.uba.ar. 
del globo, según los condicionamientos de clase, de género, étnicos y generacionales. Por lo tanto, considerar la vulnerabilidad de sujetos, grupos y comunidades remite a analizar los procesos de construcción de la vulnerabilidad psicosocial.

Ayres, França Júnior, Junqueira Calazans y Saletti Filho (2003/2008) indican que dichos procesos implican considerar el interjuego entre un componente individual (como capacidad emocional y simbólica); un componente social (como relación con los otros sociales) y un componente programático (como disponibilidad y acceso a la protección de las políticas sociales). De esta manera, la existencia de comunidades, grupos y/o sujetos vulnerables o en situación de vulnerabilidad psicosocial supone considerarlos frágiles, jurídica o políticamente, en la promoción, protección o garantía de sus derechos de ciudadanía (Di Leo \& Camarotti, 2015).

En el caso de las infancias y las adolescencias, los aspectos subyacentes a su constitución progresiva como sujetos autónomos dan cuenta de una condición de vulnerabilidad originaria. Los límites conferidos a la autorrepresentación de niños, niñas y adolescentes en la vida política, es decir, de su participación plena como ciudadanos, señalan su dependencia relativa al mundo adulto para tal ejercicio (Baratta, 1999; Lenta, 2016). Por eso, el papel que desempeñan las instituciones sociales en la producción, protección y/o tutelaje de las infancias y adolescencias resulta central en cuanto a la generación o no de espacios sociales y comunitarios para viabilizar los procesos progresivos de dicha autonomía, es decir, de participación social y desarrollo de prácticas de cuidado de sí y los otros (Carli, 2006; Llobet, 2010). En consecuencia, si en la infancia no se supera el nivel de la necesidad como mera supervivencia, los procesos de vulneración psicosocial configurarán situaciones de expulsión de la ciudadanía en el propio tiempo presente, así como también condicionarán la construcción del porvenir (Bustelo, 2011).

En este marco, la exigibilidad de los derechos de niños, niñas y adolescentes implica interpelar las situaciones de exclusión psicosocial a partir del desarrollo de dispositivos de subjetivación sostenidos desde el mundo adulto. Estos dispositivos se refieren al necesario desarrollo de espacios sociales, discursos e instituciones que se deben constituir en soportes intergeneracionales, a partir de otorgarle a las nuevas generaciones un espacio en la trama social para propiciar su individuación y constituirlos como sujetos deseantes, con la capacidad autónoma de pensarse, historiarse y proyectarse en el devenir del mundo (Agamben, 2006/2014; Bleichmar, 2009).

La exigibilidad de derechos es, entonces, un proceso social del que participan diferentes actores de la comunidad. En el caso de las infancias, esta exigibilidad impele a la promoción de la agencia de niños, niñas y adolescentes desde la protección frente a la hostilidad del mundo y la valoración de sus voces. En la habilitación de la palabra de niños, niñas y adolescentes se da lugar a la producción de sentidos y significados sobre la propia vida y el mundo, evidenciando identidades adoptadas y atribuidas, discursos sociales y relaciones de poder. A su vez, se abren posibilidades a la resignificación de la historia singular y de interpelación a los marcos culturales y sociales que constriñen el devenir de los sujetos (Stecher, 2010). En el campo de la infancia, entonces, la exigibilidad de sus derechos frente a la vulnerabilidad constitutiva y a los procesos de vulneración de sectores de niños y niñas, remite a la toma de la palabra en el sentido de de Certeau (1968/1995). Se trata del primer tiempo de la acción política y consiste en habilitar el espacio social para que los niños y niñas puedan afirmar "no soy una cosa", reclamando, así, la inscripción o reinscripción- en los dispositivos subjetivantes de la comunidad y/o las políticas sociales. En este marco, se busca que la promoción de la participación social de niños, niñas y adolescentes no solo se remita a informarlos sobre los temas de su interés de manera adecuada, a habilitar la validez de sus opiniones y a ser escuchados por el mundo adulto, sino que también implique que puedan incidir en las decisiones sobre los temas que los involucran directamente o que les interesan, poniendo en juego en ello sus capacidades cognitivas, afectivas y relacionales, como sustento para un desarrollo psicosocial pleno que garantice su lugar como ciudadanos sujetos de derechos (Giorgi, 2010; Horn, 2011; Pyerín \& Weinstein, 2015).

\section{Entre la Retórica de los Derechos y los Procesos de Vulneración de Niños, Niñas y Adolescentes}

A nivel mundial, la visibilidad y reconocimiento de los derechos humanos a partir de la segunda posguerra, mediante el desarrollo de diversos movimientos sociales, tuvo su correlato en la promulgación de tratados internacionales que, en el ámbito de la niñez, implicó la emergencia del paradigma de la protección integral de los derechos del niño en la segunda mitad del siglo XX. 
La Declaración Internacional de los Derechos del Niño (1959), las Reglas de Beijín (1985) y la Convención Internacional de los Derechos del Niño (CIDN; 1989), fueron los instrumentos fundantes en este campo. De hecho, la CIDN fue el instrumento de derechos humanos más rápidamente ratificado por los países del mundo (con excepción de Estados Unidos y Somalia). Sus aportes centrales fueron el principio del interés superior del niño, el derecho a sobrevivir y desarrollarse y la consideración de su voz en los asuntos que atañen a su propia vida.

Sin embargo, en las últimas décadas, las lógicas de ajuste fiscal de las políticas neoliberales configuraron un proceso mundial de infantilización de la pobreza, lo que pone en discusión la adscripción de los Estados a dichos tratados y, en consecuencia, el rol de las instituciones gobernadas por el mundo adulto en la garantía de los derechos de la infancia. En Argentina, esa tendencia se expresa en diversos modos de vulneración de derechos de niños, niñas y adolescentes: la tasa de pobreza en la niñez era del 46,26\% en 2013, mientras que en la población general llegaba al 31,46\% (Caggia, 2014). El deterioro de las condiciones de vida se observa también en el aumento significativo, en la última década, de la explotación laboral infantil (en talleres clandestinos), el incremento de niños y niñas viviendo en situación de calle, el aumento de la maternidad en la adolescencia temprana, el secuestro y la trata para la explotación sexual comercial infantil, entre otros (Fondo de las Naciones Unidas para la Infancia [UNICEF] \& Argentina, Ministerio de Desarrollo Social de la Nación, 2015). Estas situaciones se combinan con la crisis de las instituciones tradicionales, como la familia, el trabajo, la escuela y el hábitat, lo que "estabiliza" la precarización de la vida para franjas importantes de niños, niñas y adolescentes, en tanto no se han creado otras instituciones de cuidado (Burman, 2013; Carli, 2006; Castoriadis, 1997).

En Argentina se creó en 2005 el Sistema de Protección Integral de Derechos de Niños, Niñas y Adolescentes (SPIDNNyA), a partir de la ley 26.061 de protección integral de los derechos de niños, niñas y adolescentes. Dicho sistema constituye una matriz de principios normativos a partir de los que deben desarrollarse las políticas sociales para garantizar los derechos de las niñas, niños y adolescentes en todo el territorio argentino. Sus ejes centrales son el interés superior del niño y el enfoque de la integralidad de derechos para la infancia. En el marco anterior, los programas sociales del campo de la infancia que hacen parte del SPIDNNyA son interrogados fuertemente en cuanto a su alcance (cobertura) y eficacia en la restitución de derechos vulnerados. Y las diferentes estrategias desarrolladas por las distintas comunidades para paliar las limitaciones de las políticas sociales, como son los jardines comunitarios y los comedores populares, e incluso aquellos que se plantean como organizaciones de la sociedad civil o movimientos sociales con una perspectiva de integralidad de los derechos en la infancia, señalan fuertes limitaciones en la garantía integral de los derechos (UNICEF, 2014).

$\mathrm{Si}$, como sugiere Wiesenfeld (2014), un propósito central de la psicología social comunitaria es aportar a los procesos de emancipación de sectores oprimidos, propiciando transformaciones psicosociales en distintas escalas, entornos y con los agentes corresponsables, el abordaje de las situaciones y procesos de vulneración de derechos de niños, niñas y adolescentes constituye un campo relevante de problemas para la interrogación crítica desde la disciplina.

En el campo de la psicología social comunitaria, un conjunto considerable de investigaciones señaladas en el estudio de Di Iorio, Lenta y Hojman (2011) han abordado las situaciones de vulneración de derechos y desventaja social de niños, niñas y adolescentes en contextos de pobreza y exclusión, pero en su mayoría han enfatizado la perspectiva de los adultos de referencia, tanto familiares como institucionales (Carbonell Blanco et al., 2015; Leale, 2005, Agosto; Salvia, Tuñón \& Poy, 2014, Diciembre; Tissera, 2010, Noviembre), dejando por fuera la voz de niños, niñas y adolescentes. Incluso en estudios de evaluación de políticas sociales para las infancias, se prioriza la voz de los adultos expertos o referentes de las instituciones frente a la de los sujetos infantiles (Casas, 2010; Giorgi, 2012; Herrera-Seda \& Aravena-Reyes, 2015; Llobet, 2009; Luciani Conde, Macías, Magistris, 2015; Olivares \& Reyes-Espejo, 2016; Pelatelli \& Naumann, 2014, Julio; Torricelli, 2014, Noviembre; Tuñón, Laiño \& Castro, 2014) o de los adultos familiares (Denegri, Aguirre \& Salinas, 2015, Noviembre; Marotta, 2015).

Otras investigaciones que, en cambio, sí recuperan la voz de la población infantil y/o adolescente, circunscriben los estudios sobre la vulnerabilidad psicosocial a temáticas como la sensación de bienestar en la comunidad (Castellá Sarriera, Ferreira Moura, Morais Ximenes, \& Lopes Rodrigues, 2016), la prevención de delincuencia juvenil (Negrón Cartagena \& Serrano García, 2016), la sexualidad, el abuso sexual infantil, el embarazo en la adolescencia (de Lucio, 2004, Octubre; Zaldúa \& Pawlowicz, 2011; Zicavo, 2008, Agosto;), las trayectorias educativas vulnerables (Legaspi et al., 2009, Agosto; Lenta, Pawlowicz \& Moschella, 2016) 
LENTA Y ZALDÚA

o las situaciones de institucionalización frente a la ausencia de cuidados parentales (Di Iorio, 2009; Gueglio Saccone \& Seidmann, 2015, Noviembre), entre otros temas. Asimismo, algunos estudios vinculan estrechamente a la pobreza material con las limitaciones de los propios chicos y chicas en la proyección del futuro (Morais Ximenes \& Camurça Cidade, 2016; Tomasini, López, Bertarelli \& García Bastán, 2010, Noviembre). Sin embargo, estos estudios no abordan desde el enfoque de la psicología social comunitaria las posibilidades de exigibilidad de los derechos de la población infantil, a partir de los nudos críticos identificados por los propios niños, niñas y adolescentes habitantes de territorios de exclusión social que participan de dispositivos sociales de restitución de derechos enmarcados en políticas sociales.

Por lo tanto, este trabajo tuvo como objetivo analizar los sentidos y significados sobre sus condiciones de vida y la situación de sus derechos que construyen niños, niñas y adolescentes que participan en programas sociales del SPIDNNyA, en el Área Metropolitana de Buenos Aires (AMBA) de Argentina, en tanto el proceso de indagación dialógica promueve procesos de enunciación del yo, en y desde lo colectivo, para contrarrestar las lógicas de control que se le imponen desde diferentes instituciones sociales a la población participante. El AMBA incluye a la Ciudad Autónoma de Buenos Aires (CABA) y parte de la Provincia de Buenos Aires (PBA). Se trata de un territorio con población urbana continua, siguiendo las líneas de comunicación ferroviaria y vial, desde la CABA hacia el interior de la PBA y a lo largo de la costa del Río de La Plata. Este territorio ocupa $2.590 \mathrm{~km}^{2}$ y tiene una población de poco más de 12.800 .000 habitantes, lo que representa al 32\% de la población total de Argentina, según el Censo Nacional de Población realizado en el año 2010 (Instituto Nacional de Estadísticas y Censos [INDEC], 2012). La región se caracteriza por concentrar zonas fabriles, zonas de provisión de servicios públicos y zonas habitacionales. A su vez, en la extensión territorial se identifican conglomerados urbanos conocidos como villas miserias, donde la población carece de servicios públicos y se encuentra con altos niveles de necesidades básicas insatisfechas.

\section{Método}

En función del problema planteado, este estudio adoptó un enfoque cualitativo de investigación cuyo objetivo es comprender la realidad a partir de su interpretación, para intentar transformarla en ese proceso (Denzin \& Lincoln, 2011/2012). Este enfoque plantea la relevancia de conocer los acontecimientos sociales en el ámbito donde ocurren y ubica al investigador en el lugar de un bricoleur, es decir, de un ensamblador de contextos, técnicas, métodos, voces de los sujetos participantes, experiencia del investigador y las interpretaciones que realice.

A su vez, la inclusión explícita del investigador en el proceso de investigación hace hincapié en la relevancia de los procesos intersubjetivos subyacentes a la relación entre el investigador y su objeto, es decir, los sujetos sociales participantes, a partir de los cuales se produce la significación (Denzin \& Lincoln, 2011/2012). En síntesis, este enfoque señala: (a) el carácter constructivo-interpretativo del conocimiento, (b) el proceso de construcción de conocimiento en tanto proceso de comunicación y (c) la significación de lo singular en la propia producción del conocimiento (González Rey, 2008).

Desde esta mirada, el estudio se posicionó desde una perspectiva ética relacional (Dussel, 1998; Montero, 2004), donde el otro es comprendido como un sujeto con saberes propios y en una relación dialógica con el investigador, movimiento que produce cambios en diversos aspectos de la realidad social que se busca conocer.

En este marco, el estudio adoptó un diseño exploratorio-descriptivo en una modalidad de estudio de casos múltiple, constituido por casos típicos de vulneración de derechos, donde cada niño, niña o adolescente participante de los programas sociales de restitución de derechos enmarcados en el SPIDNNyA, constituyó un caso (Yin, 2009).

Conforme al enfoque cualitativo planteado, los criterios de validez del estudio estuvieron centrados en lograr: (a) la validez pragmática, la que se obtuvo a través de la explicitación a los participantes de los objetivos, propósitos, alcances y riesgos de su participación en la investigación; (b) la validez semántica, que se refiere al reconocimiento del contexto en la construcción de significaciones por parte de las investigadoras, alcanzada por el acercamiento previo a los dispositivos involucrados en la investigación, el reconocimiento de los antecedentes de los dispositivos a través de fuentes secundarias y entrevistas previas, el registro de los instrumentos implementados mediante soportes magnetofónicos y el abordaje previo de otras poblaciones de niños, niñas y adolescentes con derechos vulnerados y (c) la validez hermenéutica, lograda al elucidar las ideas previas de las investigadoras en el trabajo realizado en equipo antes y durante el proceso interpretativo (Denzin \& Lincoln, 2011/2012). 


\section{Participantes}

Los participantes del estudio fueron 16 chicos y chicas de entre 13 y 18 años, cinco varones y 11 mujeres, seleccionados de manera intencional a partir de su asistencia a dos programas sociales de restitución de derechos de niños, niñas y adolescentes (ocho participantes de un centro de día y ocho participantes de una sede del Servicio Local de Protección de Derechos del Niño, constituidos dentro del SPIDNNyA en el territorio del AMBA. La muestra fue homogénea en cuanto al territorio y la situación socioeconómica y heterogénea en cuanto al género, la edad, el tipo de derechos vulnerados (derecho a vivir en condiciones de bienestar y a un sano desarrollo integral, derecho a una vida libre de violencia y a la integridad personal, derecho a la protección de la salud y a la seguridad social y derecho a la educación) y el tiempo de participación en el programa social (entre seis meses y dos años). Se excluyeron especialmente los casos de chicos y chicas en situación de calle, los institucionalizados sin cuidados parentales y aquellos que se encontraban atravesando situaciones recientes de violencia sexual.

\section{Instrumentos}

En función del problema y objetivo planteados, los tópicos indagados a través de los instrumentos de producción de datos consideraron aspectos como identidad personal y colectiva, vínculos de referencia, sentidos sobre el territorio, proyecto a futuro e identificación de derechos vulnerados y a restituir.

Los instrumentos implementados para la producción de datos fueron de tipo cualitativo, lo que resulta coherente con el diseño propuesto, favoreciendo la producción individual y colectiva de narraciones orales, escritas y gráficas. Fueron los siguientes:

Relato de vida. Consiste en la construcción de un relato autobiográfico construido de modo recursivo a partir de sucesivas entrevistas en profundidad. El participante, en función de ciertos temas propuestos por el/la investigador/a, construye una narrativa retrospectiva, indicando punto de viraje o epifanías en la trayectoria de vida (Chase, 2011/2015). Según Ricoeur (2008), en este proceso el sujeto que narra lo hace desde una identidad narrativa que se construye y reconstruye mediante los relatos que dan sentido a las acciones y a los eventos vividos, permitiendo otorgar un sentido global, aunque transitorio, sobre la propia existencia. En este estudio se trabajó a partir de la construcción de relatos orales combinados con narraciones escritas y gráficas (Seidmann, Di Iorio, Azzollini \& Rigueiral, 2014), lo que favoreció aprehender distintos aspectos de la reconstrucción biográfica de cada participante.

Grupo focal. Los grupos focales consisten en grupos de discusión entre participantes con ciertas características homogéneas que permiten producir narrativas polifónicas acerca del problema social estudiado, al mismo tiempo de identificar tensiones, conflictos y consensos dentro de los grupos, favoreciendo la consolidación de identidades colectivas (Kamberelis \& Dimitriadis, 2011/2015). En la presente investigación, los grupos focales estuvieron conformados entre seis y siete participantes y las distintas sesiones abordaron las temáticas del territorio, el cuerpo y el conocimiento de los derechos, trabajando con elaboraciones narrativas orales y gráficas (Seidmann et al., 2014).

Observación naturalista. Se trata de una perspectiva de la observación que asume una posición decolonial respecto de las prácticas de investigación, superando la tensión observación-participación en el trabajo de campo. Señala la importancia de la reflexividad del investigador en torno a su género, clase, etnicidad, entre otros, como parte de la interpretación del producto etnográfico de la observación (Angrosino, 2011/2015). La misma se realizó a través de la construcción de un cuaderno de campo que incluyó el registro de las actividades, narrativas significativas, dinámicas y climas grupales en cada una de las actividades realizadas, así como también de la resignificación de las actividades realizadas y de la implicación de las propias investigadoras en el desarrollo de las actividades pautadas.

\section{Procedimiento}

En función del artículo 12 de la CIDN acerca del derecho a voz y a ser escuchado, así como del principio de autonomía progresiva de los niños, niñas y adolescentes, se trabajó con un consentimiento informado (Cornejo, Mendoza \& Rojas, 2008) suscrito por los mismos, estableciendo un contrato escrito que incluyó: contextualización y objetivos de la investigación, tipo de participación requerida y derechos del participante (voluntariedad de la participación, anonimato, confidencialidad de los datos). Cabe señalar que el mismo 
fue presentado y discutido en forma oral con cada grupo de niños, niñas y adolescentes, para luego ser refrendado de manera escrita en un espacio individual en donde se pudieron esclarecer diferentes interrogantes presentados por los mismos. También se solicitó consentimiento de al menos un tutor o responsable de cada niño, niña y adolescente y consentimiento institucional. El diseño de los instrumentos de consentimiento informado fue elaborado según la Declaración de Helsinki (actualización 2013), el código de conducta de la Asociación de Psicólogos de Buenos Aires y el Comité de Conductas Responsables en Investigación de la Facultad de Psicología de la Universidad de Buenos Aires.

El trabajo de campo se llevó a cabo entre marzo de 2014 y junio de 2015 en diferentes fases. En un primer momento, se procedió al contacto con los programas de referencia y se realizaron actividades generales de familiarización con el territorio y la dinámica de los espacios, a través de la participación en diferentes instancias institucionales (entrevistas informales, eventos y talleres con niños, niñas y adolescentes). En un segundo momento, se elaboró participativamente (con las instituciones y los niños, niñas y adolescentes) un plan de actividades acorde tanto con los objetivos del estudio como con los intereses y necesidades institucionales y de los sujetos participantes. Para ambas instituciones se planteó el desarrollo de seis grupos focales sobre los tópicos de indagación señalados anteriormente y otros afines no analizados en este estudio, organizados a través de dinámicas lúdicas, de tres horas de duración y con frecuencia mensual. Todos los niños tuvieron entre cuatro y seis participaciones en los grupos focales. A su vez, según disponibilidad para la participación y con el acuerdo del equipo técnico institucional, se realizaron entrevistas biográficas en profundidad (entre dos o tres, según el caso) antes o después de la realización de los grupos focales, al comienzo del segundo momento del estudio. Finalmente, en un tercer momento, se procedió a realizar un cierre del proceso de investigación, a través de la elaboración de un informe audiovisual para cada grupo de participantes de cada institución, con las principales producciones de las distintas instancias, el que fue compartido con los participantes del estudio. Con todos los sujetos participantes se trabajó en la construcción del relato de vida.

\section{Estrategia de Análisis de Datos}

Para el abordaje analítico de los datos se hizo énfasis en los aspectos narrativos y de producción de sentido, más que en el alcance de la dimensión participativa del proceso, en función del objetivo propuesto. Por lo tanto, para el tratamiento de los datos se apeló a una estrategia de enfoque biográfico que señala cómo la trayectoria de vida singular se construye a través de mediaciones, constituidas por los grupos primarios a los cuales se pertenece y las organizaciones comunitarias con las que se relaciona. De esta manera, se propendió a la desprivatización de la experiencia personal para dar cuenta de la experiencia como dimensión colectiva. Por ello, se priorizó una exégesis transversal de las narrativas de los participantes por sobre la exégesis singular, a partir de tres dimensiones analíticas propuestas por Bertaux (1997/2005): la realidad socio-histórica, la realidad psíquica y la realidad discursiva.

Específicamente, a partir de la audición de las grabaciones de cada uno de los encuentros individuales (relato de vida) y colectivos (grupos focales), así como de la sistematización de las observaciones, se procedió a la lectura transversal de la información mediante la asistencia del software Atlas.ti versión 7.1.0. (Friese, 2013). En un primer momento, se descartó el análisis intracaso y se desarrolló una codificación de las narrativas, a partir de los tópicos que se plantearon indagar con cada instrumento, junto con una codificación abierta de las categorías emergentes de los datos y no consideradas previamente. En un segundo momento, se procedió a una recodificación de la información mediante integración y redefinición de las categorías de la primera fase. En un tercer momento, se vincularon las categorías definidas con las dimensiones de análisis propuestas por Bertaux (1997/2005) para el análisis transversal de las narrativas.

\section{Resultados}

Para el caso de la realidad socio-histórica, comprendida como aquella dimensión que integra el enlace entre el momento histórico-social y los acontecimientos biográficos (Bertaux, 1997/2005; Kornblit, 2007), las narrativas señalaron huellas de la crisis capitalista y las políticas neoliberales de exclusión y segmentación social en la vida cotidiana de los participantes. Las situaciones de pobreza estructural y precarización del trabajo de familiares y adultos referenciales aparecen como situaciones naturalizadas que se asocian con el territorio:

[1] El barrio de los quilomberos [alborotadores], villeros nos dicen. Pero somos los más piolas [simpáticos] (...) Trabajan de rochos [ladronzuelos], trabajan de changas [trabajo esporádico], pero se trabaja. (Carlos, 14 años, grupo focal) 
[2] Casas, casa y gente. Casas y gente todo el día al pedo [no realizar actividades productivas]. Ahora unas canchitas. Todos... no hacen nada. Hay transas [que venden drogas] y narcos. (Julián, 15 años, grupo focal)

[3] Mi papá trabaja, siempre trabajó de [vendedor] ambulante. Mi mamá se mantiene de trabajar con la asignación [universal por hijo, plan social] de nosotros. (Celina, 16 años, relato de vida)

Como saturan las narrativas, al describir tanto el territorio barrial como al hacer referencia a las ocupaciones de familiares, en los relatos de los participantes, los trabajos de los adultos dan cuenta de situaciones de informalidad y precarización laboral [1] [3], así como de inserciones ocupacionales violentas [1] [2], lo que da cuenta de los efectos del modelo neoliberal que, a partir de la década de 1970, impactó sobre la organización del trabajo asalariado, expulsando a vastos sectores de la población argentina a la desocupación, la que creció exponencialmente en la década de 1990 y comienzos del 2000 (Svampa, 2005).

Asimismo, las narrativas visibilizan una territorialidad connotada como precaria y marginalizada, en relación a otros núcleos urbanos:

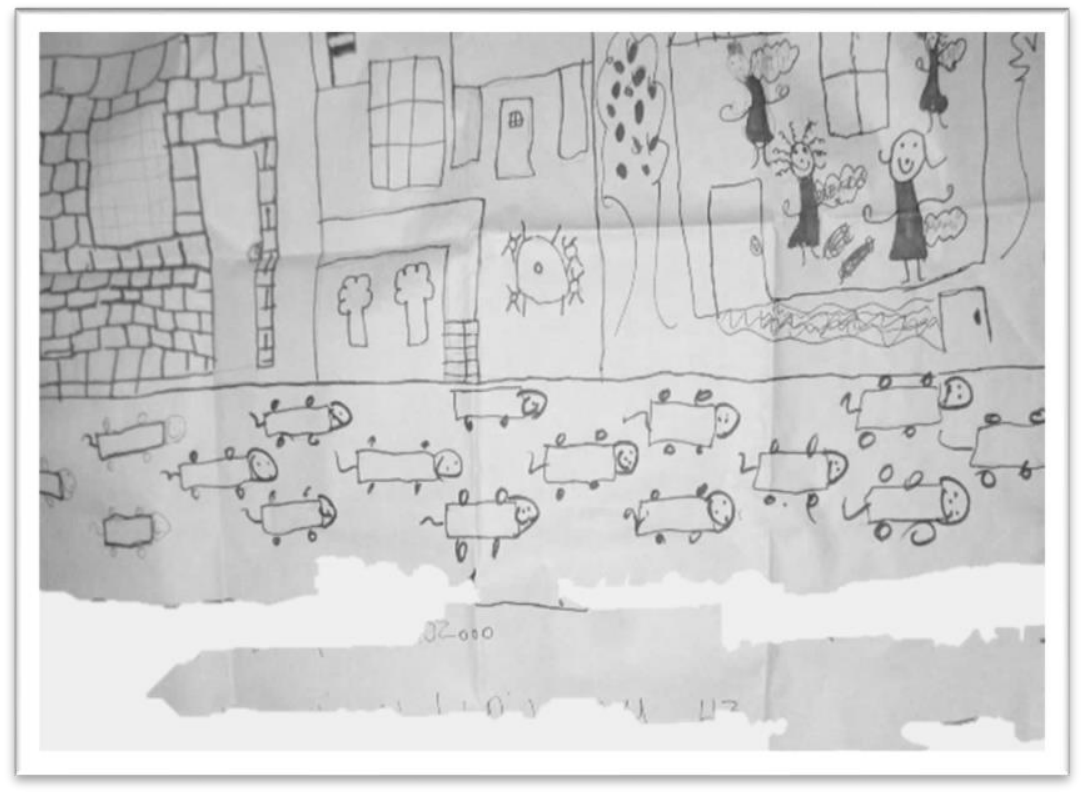

[4] Figura 1. Gráfico sobre el barrio (grupo focal, elaboración colectiva)

[5] Somos los cuatro hermanos y mi papá ahora en la casa. Tenemos la pieza y la cocina. Todavía el baño, afuera. El piso ya lo va a poner. (Magali, 16 años, relato de vida)

[6] Ni colectivos, ni cloacas, solo casa, chicos y ratas. La gente está, es buena y es mala. Casas de material, de chapa y madera. Casi no hay árboles. (Lucas, 16 años, grupo focal)

[7] Es un barrio recoleto [hace referencia a un barrio lujoso de Buenos Aires] [risas]. Le decimes recoleto porque se quiere hacer el cheto [de mayor nivel socioeconómico]. Algunos. Hay gente mucha. Es común y cualquiera. Nos cuidamos entre todos. Hay un poco de rochos [ladronzuelos]. (Julieta, 13 años, grupo focal)

El hacinamiento en las viviendas [5], la cercanía con basurales, la falta de obras públicas de saneamiento y la contaminación ambiental [4] [6] son las características sobresalientes que identifican los participantes en sus territorios. Se trata de indicadores socioambientales de exclusión social. El registro de la marginalización territorial-poblacional se expresa bajo la "guetificación" (nosotros-los otros), concomitante a las dinámicas de "gentrificación" de los territorios urbanos (Zaldúa, Bottinelli, Longo, Sopransi \& Lenta, 2016) y señala la brecha social entre territorios, indicando dinámicas de vulneración de sus pobladores [7]. No obstante, este registro aparece compensado por las prácticas de solidaridad y cuidado entre los habitantes del propio espacio [7].

La dimensión de la realidad psíquica se refiere al espacio en el que se inscriben las variantes del ser y el hacer de cada sujeto (Bertaux, 1997/2005; Kornblit, 2007). En el análisis de las narrativas acerca de la vida 
cotidiana y las trayectorias vitales, las afectaciones de las violencias emergieron como núcleo organizador de los padecimientos, el malestar y los anhelos. La violencia estructural significada en la realidad sociohistórica expresa desdoblamientos sobre los vínculos relacionales-familiares y sobre sí mismos:

[8] Me arrebataron el derecho de poder crecer con felicidad, pero no pudo ser posible eso. Maltrataron a mis hermanos, golpearon e hicieron maltrato psicológico y daño a su cuerpo, les hicieron cicatrices muy feas y rompieron el sueño de poder crecer sin miedo. (Marisa, 17 años, grupo focal)

[9] Mi mamá me dijo que haber nacido fue un error de ella y yo pensaba lo mismo. Pero yo tenía derecho a vivir bien. (Laura, 13 años, grupo focal)

[10] Finalmente mi mamá se fue. Ya no nos pega. Pero igual la extraño. Sueño que vuelve y todo está bien. (Julieta, 13 años, relato de vida)

Las situaciones de vulneración de derechos por los que estos niños, niñas y adolescentes participan en los programas sociales del SPIDNNyA son reconocidos por ellos mismos como acontecimientos y situaciones de maltrato [8] [9] y abandono [10] por parte del mundo adulto, especialmente por aquellos del ámbito familiar, lo que conjuga angustias y tristezas, pero también son reconocidas como situaciones de injusticia [8] [9], lo que instala el horizonte de los derechos como posibilidad de exigibilidad de reconocimiento social.

En algunos casos, la carencia de mediadores afectivos, como la ternura y el lazo social, propician elaboraciones fallidas y autodestructivas, como se observa en las narrativas [11] [13] y en las marcas de cortes en los brazos de la Figura 2 [12]:

[11] (...) cuando estoy sola, no hago nada. Me siento más sola de lo que estoy. Me quiero morir, me quiero matar. Necesito a alguien que me escuche para no hacerme daño yo sola. (Laura, 13 años, relato de vida)

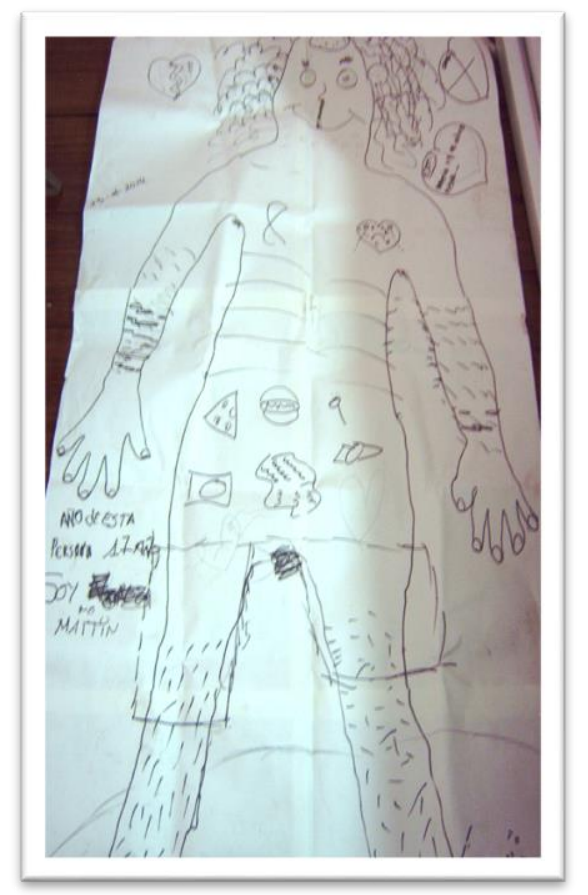

[12] Figura 2. Gráfico sobre el cuerpo (grupo focal, elaboración colectiva)

[13] Es Andrés, tiene 15 años. Le gusta la comida chatarra. Está triste. Tiene el corazón roto (...) en los brazos se cortó, no lo puede evitar. Cuando está solo, nadie lo quiere, quiere morir y se corta, se marca por el dolor. (Lucio, 15 años, relato de vida)

Sin embargo, existen otras posibilidades frente a la ausencia de protección ante las amenazas del entorno de estos niños, niñas y adolescentes, cuando la ternura y la esperanza se instalan desde las 
instituciones que emergen en los territorios de manera autogestante y con el apuntalamiento de diferentes referentes comunitarios:

[14] Sandra está ayudando a preparar mis 15. Hace lo que mi familia no hace. Sabe los problemas míos. Me tiene en cuenta. El comedor es el lugar. Me siento que me cuidan y que me quieren ahí (...) Te dan ganas de ponerte bien, de quererte. (Paula, 14 años, grupo focal)

[15] Lo bueno fue empezar primer año de la escuela. Ahí tuve nuevos amigos. Todo un cambio de hacer nuevas cosas, que no sea siempre lo mismo. Empecé a pensar cosas más buenas. (Damián, 17 años, relato de vida)

El espacio comunitario y autogestante del comedor barrial [14] cumple una función no solo de garantizar el derecho a la alimentación, sino que también se ofrece como un ámbito de sostén intergeneracional reparador de las relaciones fallidas con otros adultos, especialmente del ámbito familiar. La escuela [15] igualmente convida a la salida exofamiliar que permite reorganizar la cotidianidad desde otras lógicas, proyectos y vínculos (Quintal de Freitas, 2008).

El plano de la realidad discursiva alude al encuentro con el otro, en tanto referentes e instituciones que devuelven una interpretación sobre la trayectoria de vida de los sujetos narradores y permiten realizar reinterpretaciones retrospectivas y prospectivas acerca de lo vivido, lo fantaseado y lo imaginado (Bertaux, 1997/2005; Kornblit, 2007):

[16] Estudio para llegar a ser maestra. 2017 voy a seguir estudiando. Voy a salir a bailar, si me dejan, que ya creo que sí. 2020 voy a ser maestra de fisicoquímica. 2023 voy a decidir tener hijos y darles un futuro. Me quiero casar. Sandra me va a ayudar y yo la voy a ayudar a ella con el comedor. (Julieta, 13 años, relato de vida)

[17] Estudiar hasta 2018. Después trabajar. Después formar una familia en 2020. Tener un hijo y una hija.

Seguir acá [en el programa social]. (Julián, 15 años, relato de vida)

En este estudio, las narrativas de las instituciones de sostén extrafamiliares [17] (escuela, comedor, programa social) y de los principales referentes adultos [16] (Sandra) se configuraron como puntales identificatorios subjetivantes que permitieron imaginar proyectos futuros de no-exclusión. Los proyectos de estudio y trabajo, de cuidado de las futuras generaciones y de apropiación de los espacios comunitarios en los que los niños, niñas y adolescentes pueden agenciarse, señalan caminos posibles de restitución de derechos a partir del fortalecimiento del entramado social, aun frente a las violencias estructurales, simbólicas e intersubjetivas, en el sentido de lo que Žižek (2008/2009) denomina como trípode de las violencias y que, a su vez, amenazan en la exacerbación de los procesos de vulneración de las trayectorias de vida.

\section{Discusión y Conclusiones}

En esta investigación, las validaciones pragmáticas, semánticas y hermenéuticas se desplegaron en las formas y funciones de los recursos narrativos biográficos. Mediante el bricolage de múltiples herramientas gráficas, orales e interaccionales y de artefactos culturales y sociales, se consideró al entorno sociocultural estructural — semiótico - y a las dimensiones de la subjetividad para llevar adelante esta investigación, en consistencia con la matriz interpretativa propuesta desde la psicología social comunitaria (Montero, 2004; Wiesenfeld, 2016). Sin embargo, este enfoque presenta la limitación de que la generalización de los resultados se restringe a la población participante del estudio y resultaría pertinente extenderlo a otras poblaciones similares de niñas, niños y adolescentes con derechos vulnerados para elaborar conclusiones de mayor cobertura.

No obstante, el trabajo intensivo con los casos permitió acceder a la complejidad del fenómeno estudiado puesto que el ejercicio de reconstrucción de la biografía singular y colectiva interpela las inflexiones e insistencias de exclusión en las trayectorias de vida de niños, niñas y adolescentes en situaciones de vulnerabilidad psicosocial. Esta modalidad propicia la dialogicidad y la reinterpretación de acciones o eventos en un proceso de producción de narrativa identitaria (Hall, 1996/2003; Ricoeur, 2008) de los sujetos participantes de programas sociales de restitución de derechos.

Las autopercepciones de discriminación de los otros a través de la mirada o los actos y los atributos devaluados de sus territorios de pertenencia, constituyen un registro de las diferencias singulares y colectivas marcadas en sus cuerpos y discursos. Las violencias sociales estructurales (precarización del trabajo, violencia social y contaminación del hábitat) se conjugan con la crisis de las instituciones familiares, en las que el sostén intergeneracional falla. La violencia física, el abandono y los abusos configuran núcleos significativos de vulneración de derechos, que se reactualizan como violencias sobre sí mismos. Estos diferentes procesos de negación del reconocimiento producen diferentes procesos de 
vulneración afectiva, corporal, simbólica y/o relacional que delimitan ciudadanías parciales para estas infancias, aun cuando participen de programas sociales de restitución de derechos.

La violencia omnipresente —objetiva y subjetiva, en términos de Žižek (2008/2009)— que aparece en las narrativas de chicos y chicas opera fragilizando las existencias, denegando posibilidades de sostén en los vínculos primarios (de referentes familiares) y propiciando procesos desubjetivantes. Es decir, la violencia en sus distintas dimensiones, social, simbólica y directa (maltrato, abandono, entre otros), impele a estados de mera supervivencia (en el sentido material y psicosocial), cuando la falta de vínculos intersubjetivos en el presente constriñe e, incluso, clausura la fantasía sobre lo "porvenir", o sea, en la proyección de los chicos y chicas hacia un futuro diferente al de la violencia y la exclusión.

Los casos presentados cuestionan la eficacia de las políticas sociales con las infancias y adolescencias en los territorios marginalizados. Los dispositivos abordados de restitución de derechos intentan promover proyectos de vida a partir de la habilitación de discursos sociales de reconocimiento hacia las infancias y adolescencias excluidas, pero sin incidir en las violencias estructurales y operando de manera acotada en la reconstitución o constitución de lazos familiares de sostén intergeneracional. En cambio, se observan estrategias de reconstrucción del lazo social, considerando la apuesta a otras instituciones de la comunidad que posibilitan otros soportes subjetivantes. El centro de día, la escuela y el comedor pudieron ser visibilizados como dispositivos que promueven la exigibilidad y el acceso progresivo a los derechos, en la medida en que los chicos y chicas pueden reconocerse intersubjetivamente en un "soy" con el otro. En estos espacios microsociales, los abandonos parentales, las crisis familiares y las fantasías compensatorias que aparecen en algunos relatos de vida y en la producción colectiva de los grupos focales, son resignificados mediante el reconocimiento de otros pares y adultos. En términos de Honneth (1994/1997), este reconocimiento no solo se da en la esfera intersubjetiva del amor o la ternura cuando los espacios permiten desarrollar prácticas de cuidado, sino que los espacios mencionados incluyen a la esfera de la solidaridad social al incluir referentes comunitarios que implican otros referentes identificatorios para los jóvenes y también, incluyen a la esfera de los derechos como parte de las dinámicas del reconocimiento. Pues desde allí se interpelan al quehacer de los programas sociales y las instituciones, como la escuela, en cuanto a la garantía de derechos para esta población.

El cuidado y reconocimiento de sí por otros significativos, la ternura como afecto frente a la hostilidad y mortificación habitual de sus trayectorias y la posibilidad de habilitar/habitar espacios de formación, capacitación y encuentro, son tres dimensiones que permiten imaginar proyectos y esperanzas. Allí donde los espacios familiares no constituyen una trama de sostén subjetivante y donde las dinámicas de las políticas de exclusión social compelen al aislamiento y la estigmatización, estas dimensiones aparecen como posibles caminos para la exigibilidad de los derechos, desde estos dispositivos de programas sociales y organizaciones comunitarias que se posicionan en la perspectiva de protección integral de derechos para las infancias.

\section{Recomendaciones}

Desde la psicología social comunitaria, considerar las trayectorias de vulneración de niños, niñas y adolescentes en la perspectiva de exigibilidad de sus derechos, plantea como desafíos:

- Valorar los registros epidemiológicos cuantitativos de niños, niñas y adolescentes en situación de vulnerabilidad, pero también contribuir, desde una perspectiva intensiva-cualitativa, a reconocer las trayectorias vitales en los contextos socio-históricos donde acontecen.

- Dar lugar a la voz de los niños, niñas y adolescentes como sujetos con capacidad de reflexionar y tomar decisiones acerca de sus propias vidas y, por lo tanto, de incidir en el diseño y la dinámica de las políticas y los programas sociales en los que participan.

- Identificar y promover programas sociales y dispositivos comunitarios alternativos o sustitutivos a la lógica tutelar, que habiliten la dialogicidad desde un enfoque ético, basado en la relación, y que consideren a los niños, niñas y adolescentes como sujetos de derecho.

\section{Referencias}

Agamben, G. (2006/2014). ¿Qué es un dispositivo? Seguido de El amigo y La Iglesia y el Reino (M. Ruvituso, Trad.; Título original: Che cos'e un dispositivo?; L'amico; La Chiesa e il Regno). Buenos Aires, Argentina: Adriana Hidalgo.

Angrosino, M. V. (2011/2015). Recontextualización de la observación (E. Méndez, Trad.; Título original: Recontextualizing Observation: Etnogaphy, Pedagogy, and the Prospects for a Progressive Political Agenda). En N. K. Denzin \& Y. S. Lincoln (Coords.), Manual de investigación cualitativa. Volumen IV: métodos de recolección y análisis de datos (E. Méndez, Trad.; Título 
original: The Sage Handbook of Qualitative Research. Part IV: Methods of Collectings and Analyzing Empirical Material) (pp. 203-234). Barcelona, España: Gedisa.

ATLAS.ti (7.1.0.) [Scientific Software]. Berlin: GmbH.

Ayres, J. R. C. -M., França Júnior, I., Junqueira Calazans, G. \& Saletti Filho, H. C. (2003/2008). El concepto de vulnerabilidad y las prácticas de salud: nuevos desafíos y perspectivas (C. García de la Santa, Trad.; Título original: O Conceito de Vulnerabilidade e as Práticas de Saúde: novas perspectivas e desafios). En D. Czeresnia \& C. Machado de Freitas (Orgs.), Promoción de la salud: conceptos, reflexiones, tendencias. (1 ${ }^{\circ}$ Reimpresión) (C. García de la Santa, Trad.; Título original: Promocao da Saúde: coinceitos, reflexoes, tendencias) (pp. 135-162). Buenos Aires, Argentina: Lugar.

Baratta, A. (1999). Infancia y democracia. En Fondo de las Naciones Unidas para la Infancia, Derecho a tener derecho: infancia, derecho y políticas sociales en América Latina (Tomo 4, pp. 207-236). Montevideo, Uruguay: Editor.

Bertaux, D. (1997/2005). Los relatos de vida. Perspectiva etnosociológica (J. Monclús, Trad.; Título original: Les récits de vie). Barcelona, España: Bellaterra.

Bleichmar, S. (2009). El desmantelamiento de la subjetividad: estallido del yo. Buenos Aires, Argentina: Topía.

Burman, E. (2013). Entre dos deudas: niño y desarrollo (inter)nacional. Teoría y Crítica de la Psicología, 3, 3-19. Extraído de http://teocripsi.com/documents/3BURMAN.pdf

Bustelo, E. S. (2011). El recreo de la infancia. Argumentos para otro comienzo (2a ed.). Avellaneda, Argentina: Siglo XXI.

Butler, J. (2004/2012). Vida precaria. El poder de duelo y la violencia (F. Rodríguez, Trad.; Título original: Precararius Life. The Power of Mourning and Violence) ( $2^{\mathrm{a}}$ ed.). Buenos Aires, Argentina: Paidós.

Caggia, M. (2014). Pobreza e indigencia en niños y adolescentes de Argentina. Córdoba, Argentina: Centro de Investigaciones Participativas en Políticas Económicas y Sociales.

Carbonell Blanco, O. A. M., Plata Contreras, S. J., Bermúdez Jaimes, M. E., Suárez Baracaldo, L. C., Peña Patiño, P. A. \& Villanueva Betancourth, C. (2015). Caracterización de prácticas de cuidado en familias colombianas con niños en primera infancia en situación de desplazamiento forzado. Universitas Psychologica, 14, 67-80. https://doi.org/10.11144/Javeriana.upsy14-1.cpcf

Carli, S. (Comp.) (2006). La cuestión de la infancia. Entre la escuela, la calle y el shopping. Buenos Aires, Argentina: Paidós.

Casas, F. (2010). Representaciones sociales que influyen en las políticas sociales de infancia y adolescencia en Europa. Pedagogía Social, 17, 15-28. https://doi.org/10.7179/PSRI_2010.17.02

Castellá Sarriera, J., Ferreira Moura Jr., Morais Ximenes, V. \& Lopes Rodrigues, A. (2016). Sentido de comunidade como promotor de bem estar em crianças brasileiras [Sentido de comunidad como promotor del bienestar de los niños brasileros]. Revista Interamericana de Psicología, 50(1), 106-116. Extraído en https://journal.sipsych.org/index.php/IJP/article/view/95/pdf

Castoriadis, C. (1997). El avance de la insignificancia. Buenos Aires, Argentina: Editorial Universitaria de Buenos Aires.

Chase, S. (2011/2015). Investigación narrativa (E. Méndez, Trad.; Título original: Narrativa Inquiry: Multiple Lenses, Approach, Voices). En N. K. Denzin \& Y. S. Lincoln (Coords.), Manual de investigación cualitativa. Volumen IV: métodos de recolección y análisis de datos (E. Méndez, Trad.; Título original: The Sage Handbook of Qualitative Research. Part IV: Methods of Collectings and Analyzing Empirical Material) (pp. 58-112). Barcelona, España: Gedisa.

Cornejo, M., Mendoza, F. \& Rojas, R. C. (2008). La investigación con relatos de vida: pistas y opciones del diseño metodológico. Psykhe, 17(1), 29-39. https://doi.org/10.4067/S0718-22282008000100004

de Certeau, M. (1968/1995). La toma de la palabra y otros escritos políticos (A. Pescador, Trad.; Título original: La prise de parole et autres écrits politiques). México DF, México: Universidad Iberoamericana/Iteso.

de Lucio, A. T. (2004, Octubre). Abuso y explotación sexual infantil y adolescente. Demonización de la pobreza. En Universidad de Buenos Aires, Facultad de Ciencias Sociales, Actas de las VI Jornadas de Sociología: zpara qué la sociología en la Argentina actual? (pp. 127-132). Buenos Aires, Argentina: Editor.

Denegri, A., Aguirre, A. \& Salinas, V. (2015, Noviembre). Entramando vínculos con niñas, niños y adolescentes y con redes comunitarias. Vulnerabilidad y perspectiva de género. En V. Piatti (Comp.), Memorias del V Congreso Internacional de Investigación de la Facultad de Psicología de la Universidad Nacional de La Plata (pp. 1156-1559). Ensenada, Argentina: Editor. Extraído de http://sedici.unlp.edu.ar/handle/10915/55229

Denzin, N. K. \& Lincoln, Y. S. (Coords.) (2011/2012). Manual de investigación cualitativa. Volumen II: paradigmas y perspectivas en disputas (V. Weisntabl de Iraola, Trad.; Título original: The Sage Handbook of Qualitative Research. Part II: Paradigms and Perspectives in Contentions). Barcelona, España: Gedisa.

Di Iorio, J. (2009). Infancia e institucionalización: abordaje de problemáticas sociales actuales. Pesquisas e Práticas Psicossociais,4(2), 143-150. Extraído de https://www.ufsj.edu.br/portal2-repositorio/File/revistalapip/volume4_n2/di_iorio.pdf

Di Iorio, J., Lenta, M. \& Hojman, G. (2011). Conceptualizaciones sobre la infancia. De la minoridad al interés superior del niño. Un estudio de las producciones científicas en psicología. Anuario de Investigaciones, 18, 227-236. Extraído en http://www.scielo.org.ar/scielo.php?script=sci_arttext\&pid=S1851-16862011000100024\&lang=es

Di Leo, P. F. \& Camarotti, A. C. (2015). Introducción. En P. F. Di Leo \& A. C. Camarotti (Dirs.), Individuación y reconocimiento. Experiencias de jóvenes en la sociedad actual (pp. 9-20). Buenos Aires, Argentina: Teseo.

Dussel, E. (1998). La ética de la liberación: ante el desafío de Apel, Taylor y Vattimo con respuesta crítica inédita de K.-O. Apel. México DF, México: Universidad Autónoma del Estado de México.

Fondo de las Naciones Unidas para la Infancia (2014). Estimación y análisis del gasto público social dirigido a los niños, niñas y adolescentes en la provincia de San Juan. Buenos Aires, Argentina: Autor.

Fondo de las Naciones Unidas para la Infancia \& Argentina, Ministerio de Desarrollo Social de la Nación (2015). Encuesta sobre condiciones de vida de niñez y adolescencia Principales resultados. Buenos Aires, Argentina: Autores.

Giorgi, V. (2012). Entre el control tutelar y la producción de ciudadanía: aportes de la psicología comunitaria a las políticas de infancia. En J. Alfaro, A. Sánchez \& A. Zambrano (Comps.), Psicología comunitaria y políticas sociales: reflexiones y experiencias (pp. 201226). Buenos Aires, Argentina: Paidós.

Giorgi, V. (2010). La participación de niños, niñas y adolescentes en las Américas: a 20 años de la Convención de los Derechos del Niño. Montevideo, Uruguay: Organización de los Estados Americanos/Instituto Interamericano del niño, la niña y adolescentes.

González Rey, F. (2008). Subjetividad y psicología crítica: implicaciones epistemológicas y metodológicas. En B. Jiménez-Domínguez (Comp.), Subjetividad, participación e intervención comunitaria. Una visión crítica desde América Latina (pp. 31-54). Buenos Aires, Argentina: Paidós. 
Gueglio Saccone, C. L. \& Seidmann, S. (2015, Noviembre). El niño como sujeto de cuidado: categorías de análisis para un abordaje en representaciones sociales. En Universidad de Buenos Aires, Facultad de Psicología, Memorias del VII Congreso Internacional de Investigación y Práctica Profesional en Psicología, XXII Jornadas de Investigación y XI Encuentro de Investigadores en Psicología del MERCOSUR (Tomo I, pp. 96-99). Buenos Aires, Argentina: Editor.

Hall, S. (1996/2003). Introducción: ¿quién necesita "identidad"? (H. Pons, Trad.; Título original: Introduction: Who Needs "Identity"?). En S. Hall \& P. du Gay (Comps.), Cuestiones de identidad cultural (H. Pons, Trad.; Título original: Cuestions of Cultural Identity) (pp. 13-39). Buenos Aires, Argentina: Amorrortu.

Herrera-Seda, C. \& Aravena-Reyes, A. (2015). Imaginarios sociales de la infancia en la política social chilena (2001-2012). Revista Latinoamericana de Ciencias Sociales, Niñez y Juventud, 13, 71-84. https://doi.org/10.11600/1692715x.1312280813

Honneth, A. (1992/1997). La lucha por el reconocimiento: por una gramática moral de los conflictos sociales (M. Ballestero, Trad.; Título original: Kampf um anerkennung: Zur moralischen Grammatik sozialer Konflikte). Barcelona, España: Crítica.

Horn, A. (2011, Noviembre). La ciudadanía infantil. Un estudio sobre las ideas infantiles del derecho a la intimidad en la escuela, en Instituto de Investigaciones Gino Germani, Actas de las VI Jornadas de Jóvenes Investigadores. Eje 10: Ciudadanía, democracia, representación. Buenos Aires, Argentina: Instituto de Investigaciones Gino Germani/Universidad de Buenos Aires, Facultad de Ciencias Sociales. Extraído de https://www.aacademica.org/000-093/302.pdf

Instituto Nacional de Estadísticas y Censos (2012). Censo Nacional de Población, Hogares y Viviendas 2010 Censo del Bicentenario Resultados definitivos Serie B $N^{\circ}$ 2. Tomo 1. Buenos Aires, Argentina: Autor.

Kamberelis, G. \& Dimitriadis, G. (2011/2015). Grupos focales (E. Méndez, Trad.; Título original: Focus Groups: Contingent Articulations of Pedagogy, Politics, and Inquiry). En N. K. Denzin \& Y. S. Lincoln (Coords.), Manual de investigación cualitativa. Volumen IV: métodos de recolección y análisis de datos (E. Méndez, Trad.; Título original: The Sage Handbook of Qualitative Research. Part IV: Methods of Collectings and Analyzing Empirical Material) (pp. 494-452). Barcelona, España: Gedisa.

Kornblit, A. L. (2007). Historia y relatos de vida: una herramienta clave en metodologías cualitativas. En A. L. Kornblit (Coord.), Metodologías cualitativas en ciencias sociales: modelos y procedimientos de análisis ( $2^{\mathrm{a}}$ ed., pp. 15-33). Buenos Aires, Argentina: Biblos.

Leale, H. (2005, Agosto). Infancia vulnerable. El arte como mediador en la praxis psicosocial comunitaria en salud. En Universidad de Buenos Aires, Facultad de Psicología, Memorias de las XII Jornadas de Investigación y Primer Encuentro de Investigadores en Psicología del MERCOSUR: avances, nuevos desarrollos e integración regional (Tomo II, pp. 78-79). Buenos Aires, Argentina: Editor.

Legaspi, L., Aisenson, G., Valenzuela, V., Duro, L., Celeiro, R., De Marco, Inaebnit, V. \& Pereda, Y. (2009, Agosto). El clima escolar y el significado de la escuela para los jóvenes. En Universidad de Buenos Aires, Facultad de Psicología, Memorias del I Congreso Internacional de Investigación y Práctica Profesional en Psicología, XVI Jornadas de Investigación y Quinto Encuentro de Investigadores en Psicología del MERCOSUR (Tomo III, pp. 318-320). Buenos Aires, Argentina: Editor.

Lenta, M. M. (2016). Niños, niñas y adolescentes en situación de calle: discursos sobre la infancia y procesos de subjetivación. Buenos Aires, Argentina: Editorial Universitaria de Buenos Aires.

Lenta, M. M., Pawlowicz, M. P. \& Moschella, R. (2016). Condicionamientos sociales y de género adolescentes escolarizados desde la perspectiva de la psicología crítica. En G. Zaldúa (Coord.), Intervenciones en psicología social comunitaria: territorios, actores y políticas sociales (pp. 183-206). Buenos Aires, Argentina: Teseo.

Llobet, V. (2009). Las políticas sociales para la infancia, la psicología y el problema del reconocimiento. Investigaciones en Psicología, 14(2), 73-94. Extraído de http://www.scielo.br/pdf/fractal/v23n3/v23n3a02.pdf

Llobet, V. (2010) ¿Fábricas de niños? Las instituciones en la era de los derechos. Buenos Aires, Argentina: Centro de Publicaciones Educativas y Material Didáctico.

Luciani Conde, L., Macías, G., Pelatelli, S. \& Naumann, S. (2014, Julio). El derecho a la salud y a la alimentación en la niñez: estudios del Sistema de Protección Integral del Municipio de Lanús. En Universidad Nacional de Rosario, Actas del XI Congreso Argentino de Antropología Social (pp. 1-19). Rosario, Argentina: Universidad Nacional de Rosario, Facultad de Humanidades y Artes, Escuela de Antropología. Extraído de http://cdsa.aacademica.org/000-081/1534.pdf

Magistris, G. (2015). Del niño en riesgo al niño sujeto de derechos. Los ¿nuevos? sujetos destinatarios de los sistemas de protección de la niñez contemporáneos. Revista Niños, Menores e Infancias, 9, 55-72. Extraído de https://ar.vlex.com/vid/nino-riesgo-nino-sujeto-649851489

Marotta, C. (2015). El trabajo con familias en políticas sociales: intervención e invención en psicología social comunitaria. Psicología, Conocimiento y Sociedad, 5(2), 388-413. Extraído de http://www.scielo.edu.uy/pdf/pcs/v5n2/v5n2a15.pdf

Montero, M. (2004). Relaciones entre psicología social comunitaria, psicología crítica y psicología de la liberación: una respuesta latinoamericana. Psykhe, 13(2), 17-28. https://doi.org/10.4067/S0718-222820040002000171

Montero, M. (2006). Introducción a la psicología comunitaria: desarrollo, conceptos y procesos. Buenos Aires, Argentina: Paidós.

Morais Ximenes, V. \& Camurça Cidade, E. (2016). Juventude e pobreza: implicações psicossociais do fatalismo [Juventud y pobreza: implicaciones psicosociales del fatalismo]. Revista Interamericana de Psicología, 50(1), 128-136. Extraído de https://journal.sipsych.org/index.php/IJP/article/view/77/pdf

Negrón Cartagena, N. L. \& Serrano García, I. (2016). Prevención de delincuencia juvenil: ¿qué deben tener los programas para que sean efectivos? Revista Interamericana de Psicología, 50(1), 117-127. Extraído en https://journal.sipsych.org/index.php/IJP/article/view/43/pdf

Olivares, B. \& Reyes-Espejo, M. I. (2016). Evaluación de un programa social en infancia desde los principios de la psicología comunitaria. Revista Latinoamericana de Ciencias Sociales, Niñez y Juventud, 14, 431-444. https://doi.org/10.11600/1692715x.14129090215

Pyerín, C. \& Weinstein, M. (2015). La participación e influencia de niños, niñas y adolescentes en políticas públicas en Chile. Hacia un marco de protección integral de la niñez y adolescencia (Serie Reflexiones Infancia y Adolescencia $\mathrm{N}^{\circ}$ 21). Santiago, Chile: Fondo de las Naciones Unidas para la Infancia.

Quintal de Freitas, M. F. (2008). Red de tensiones en la vida cotidiana: análisis desde la perspectiva de la psicología social comunitaria. En B. Jiménez-Domínguez (Comp.), Subjetividad, participación e intervención comunitaria: una visión crítica desde América Latina (pp. 165-183). Buenos Aires, Argentina: Paidós.

Ricoeur, P. (2008). Hermenéutica y acción. De la Hermenéutica del Texto a la Hermenéutica de la Acción (JC Scannone, Trad.; Título original: Herméneutique et action. De l'herméneutiquedu texte a L'herméneutique de l'action, $3^{a}$ ed.). Buenos Aires, Argentina: Prometeo.

Salvia, A., Tuñón, I. \& Poy, S. (2014, Diciembre). Efectos de la asignación universal por hijo en el ingreso per-cápita familiar, en la pobreza extrema e indicadores de desarrollo humano de la infancia. En Universidad Nacional de La Plata, Facultad de Humanidades y Ciencias de la Educación, Actas de las VIII Jornadas de Sociología de la UNLP (pp. 1-31). Ensenada, Argentina: Editor. Extraído de http://www.memoria.fahce.unlp.edu.ar/trab_eventos/ev.4500/ev.4500.pdf 
Seidmann, S., Di Iorio, J., Azzollini, S. \& Rigueiral, G. (2014). El uso de técnicas gráficas en investigaciones sobre representaciones sociales. Anuario de Investigaciones, 21(1), 177-185. Extraído de http://www.scielo.org.ar/scielo.php?script=sci_arttext\&pid=S1851-16862014000100017\&lang=es

Stecher, A. (2010). El análisis crítico del discurso como herramienta de investigación psicosocial del mundo del trabajo. Discusiones desde América Latina. Universitas Psychologica 9(1), 93-107. Extraído de http://www.scielo.org.co/pdf/rups/v9n1/v9n1a08.pdf

Svampa, M. (2005). La sociedad excluyente: la Argentina bajo el signo del neoliberalismo. Buenos Aires, Argentina: Taurus.

Tissera, O. E. (2010, Noviembre). Juegoteca comunitaria y complejidad. En Universidad de Buenos Aires, Facultad de Psicología, Memorias del II Congreso Internacional de Investigación y Práctica Profesional en Psicología, XVII Jornadas de Investigación y Sexto Encuentro de Investigadores en Psicología del MERCOSUR (Tomo IV, pp. 196-198). Buenos Aires, Argentina: Editor.

Tomasini, M., López, C. J., Bertarelli, P. \& García Bastán, G. (2010, Noviembre). La pelea entre jóvenes: búsqueda de reconocimiento social en un cruce de miradas. En Universidad de Buenos Aires, Facultad de Psicología, Memorias del II Congreso Internacional de Investigación y Práctica Profesional en Psicología, XVII Jornadas de Investigación y Sexto Encuentro de Investigadores en Psicología del MERCOSUR (Tomo IV, pp. 199-201). Buenos Aires, Argentina: Editor.

Torricelli, F. (2014, Noviembre). Itinerarios de cuidado: evaluación de un programa de atención comunitaria destinado a niños, niñas y adolescentes. En Universidad de Buenos Aires, Facultad de Psicología, Memorias del VI Congreso Internacional de Investigación y Práctica Profesional en Psicología, XXI Jornadas de Investigación y Décimo Encuentro de Investigadores en Psicología del MERCOSUR (Tomo II, pp. 70-71). Buenos Aires, Argentina: Editor.

Tuñón, I., Laiño, F. \& Castro, H. (2014). El juego recreativo y el deporte social como política de derecho. Su relación con la infancia en condiciones de vulnerabilidad social. Educación Física y Ciencia, 16(1), 10-16. Extraído de https://www.efyc.fahce.unlp.edu.ar/article/view/EFyCv16n01a04/5910

Wiesenfeld, E. (2014). La psicología social comunitaria en América Latina: ¿consolidación o crisis? Psicoperspectivas, $13(2), 6-18$. https://doi.org/10.5027/psicoperspecrivas-Vol13-Issue2-fulltext-357

Wiesenfeld, E. (2016). Trascendiendo confines disciplinares: continuidad, psicología comunitaria crítica y psicología social comunitaria al revés. Revista Interamericana de Psicología, 50(1), 4-13. Extraído de https://journal.sipsych.org/index.php/IJP/article/view/65/pdf

Yin, R. K. (2009). Case study research: Design and methods ( $4^{\mathrm{a}}$ ed.). Thousand Oaks, CA: SAGE.

Zaldúa, G., Bottinelli, M. M., Longo, R., Sopransi, M. B. \& Lenta, M. M. (2016). Exigibilidad y justiciabilidad desde la epidemiología territorial. En G. Zaldúa (Coord.), Intervenciones en psicología social comunitaria: territorios, actores y políticas sociales (pp. 2154). Buenos Aires, Argentina: Teseo.

Zaldúa, G. \& Pawlowicz, M. P. (2011). Representaciones sociales de la maternidad adolescente. En G. Zaldúa (Coord.), Epistemes y prácticas de psicología preventiva (pp. 163-176). Buenos Aires, Argentina: Editorial Universitaria de Buenos Aires.

Zaldúa, G., Pawlowicz, M. P., Longo, R., Sopransi, M. B. \& Lenta, M. M. (2016). Vulneración de derechos y alternativas de exigibilidad en salud comunitaria. En G. Zaldúa (Coord.), Intervenciones en psicología social comunitaria: territorios, actores y políticas sociales (pp. 55-86). Buenos Aires, Argentina: Teseo.

Zicavo, E. (2008, Agosto). Aspectos culturales del embarazo y maternidad adolescente en los sectores populares: identidades, prácticas, representaciones. En Universidad Nacional de Misiones, Facultad de Humanidades y Ciencias Sociales, Actas del IX Congreso Argentino de Antropología Social (pp. 1-8). Posadas, Argentina: Editor.

Žižek, S. (2008/2009). Sobre la violencia. Seis reflexiones marginales (AF Antón Fernández, Trad.; Título original: Violence). Buenos Aires, Argentina: Paidós.

Fecha de recepción: Junio de 2017.

Fecha de aceptación: Octubre de 2019. 